\title{
EL ADULTO MAYOR AL FINAL DE LA VIDA: PERSPECTIVA BIOÉTICA
}

Luis Vólmar Quintero Pacheco MD*

\section{Resumen}

La vejez como situación cada vez más frecuente y compleja en nuestra época, ha sido objeto de múltiples estudios desde diferentes puntos de vista. Una mirada amplia nos muestra tres hechos clave que inciden en esta situación: el envejecimiento poblacional, el avance técnico-científico y la aparición de la bioética, que con su análisis metodológico propio logra ampliar la comprensión de los dilemas característicos de esta etapa de la vida, como son el replanteamiento de los objetivos de los tratamientos ofrecidos por el personal de salud, los cambios en la relación médico-paciente y la evaluación de la competencia mental para la toma de decisiones.

Palabras clave: vejez, final de la vida, bioética.

\section{THE ELDERLY AT THE END OF LIFE: A BIOETHICAL PERSPECTIVE}

\section{Abstract}

Old age as a more frequent and complex situation in our time has been the topic of many studies conducted from different standpoints. A comprehensive overview reveals three key factors which have implications for this matter: population aging, technical and scientific breakthroughs and the emergence of bioethics, which may broaden, through its own methodological analysis, the understanding of the typical dilemmas of this stage. These dilemmas may be: the redefinition of the purpose of treatments offered by healthcare professionals, changes in physician-patient relationship and assessment of mental competence for decision-making.

Key words: old age, end of life, bioethics.

\section{Presentación}

La primera situación que uno observa cuando se trata de mencionar a este grupo poblacional tan particular es la cantidad de eufemismos existentes no solo en la literatura seria sino en el lenguaje popular; algunos resaltan las cualidades de esas personas como "adulto mayor, decano, veterano, patriarca, venerable, etc".
Pero otros son burlones o en forma clara ofensivos, como "vejete, viejo, añejo, cucho, catano o fósil". No puedo dejar de mencionar "paciente geriátrico", como si todas las personas que están en esa edad fuesen siempre enfermas.

En segundo lugar es muy importante diferenciar dos conceptos que aunque tienen que ver muy de cerca
Fecha recibido: junio 18 de 2008 - Fecha aceptado: agosto 4 de 2008

* Especialista en Psiquiatría, Universidad del Rosario, Especialista y Magíster en Bioética, Universidad El Bosque. Presidente Comité de Ética en Investigación, Universidad El Bosque, Miembro del Comité Provincial de Bioética, OHSJD. Ex-Director Científico Clínica San Juan de DiosChía. Profesor Asistente Salud Mental y Bioética y director Oficina de Orientación Universitaria, Fundación Universitaria de Ciencias de la Salud, Bogotá, D.C. Colombia. 
uno con otro, son diferentes. Cuando hablamos de vejez debemos entenderlo como el estado histórico social de una persona; a su vez, envejecimiento es el proceso paulatino de pérdida de capacidades. Lo que deseo resaltar de esto es que toda persona tiene su propia percepción de cada uno de ellos y que son situaciones estrictamente individuales.

Podemos destacar tres hechos importantes en relación con el tema que nos ocupa:

1. Envejecimiento poblacional: deseo poner en relieve algunos elementos que están soportados en la literatura. Para el año 2020 el 16\% de la población será mayor de 60 años, mientras que el 7.5\% tendrá más de 65 años. Esto como es natural tiene que ver con el aumento de las tasas de natalidad, lo mismo que el incremento de la esperanza de vida, que supera en promedio los 70 años; la mayoría de estas personas serán mujeres y las causas de muerte las enfermedades cardiovasculares, el cáncer, las afecciones degenerativas y con un preocupante incremento, las complicaciones de las afecciones mentales.

Este aumento trae sus consecuencias: se observarán mayores tasas de discapacidad sensorial, motora y mental; los ancianos en promedio cursan con tres a cuatro enfermedades crónicas no terminales de manara simultanea; además, se suma el hecho que ellos son considerados no productivos económicamente y aún más como una carga para la sociedad, con una situación no menos lamentable como es su soledad $\mathrm{y}$ abandono.

Este proceso denominado envejecimiento tiene tres características: es heterogéneo, es decir no todo tiene la misma velocidad, es individual y no es universal. Es bastante común tomar como parámetro los 65 años para determinar que una persona ha pasado a la tercera edad; esto es probable que tenga gran utilidad para los estudios estadísticos y demográficos, pero por ello no deja de ser una norma arbitraria.

La geriatría como especialidad médica está dirigida al individuo y no a su enfermedad, lo cual tiene una estrecha relación con lo mencionado arriba: el hecho de ser anciano no es equivalente a estar enfermo; además, se enfoca a la prevención y si es posible a la curación de las condiciones patológicas que deterioran la salud; está basada en la funcionalidad global de la persona y tiene como elemento fundamental que en todo este proceso se debe involucrar a la familia, con el objeto de dar al anciano soporte emocional para la toma de decisiones y en los cuidados generales que requiere. Es necesario mencionar también que el proceso de desvalimiento cognitivo, motor, afectivo y social se encuentra presente en mayor o menor grado y es progresivo e irreversible.

Muchos de los profesionales de las ciencias de la salud con frecuencia ven como enemigos a la vejez y la muerte, negando estas realidades con su omnipotencia, lo que ha producido grandes debates sobre el papel que deben jugar en estas últimas etapas de la vida de sus pacientes. Por otra parte, vemos también que muchas sociedades en la teoría ensalzan la vejez, resaltando los valores de la experiencia y sabiduría, pero por otro lado en la práctica se considera como una pérdida.

2. El avance técnico-científico: nadie pone en duda que se ha logrado en las últimas épocas un crecimiento nunca antes imaginado, en especial en las ciencias de la salud, con sus incuestionables beneficios para la humanidad. Podría mencionar algunas de las más importantes consecuencias: el avance del conocimiento nos ha proporcionado una inmensa avalancha de información; por obvias razones la medicina ha tenido que superespecializarse. No podría dejar de mencionar el cambio dramático de la relación médicopaciente desde un modelo netamente paternalista, vigente por muchísimos siglos en donde la posición del paciente era aplastada por la del médico, para dar paso al modelo donde impera la autonomía de aquel y que se acompaña de las nuevas tendencias en los modelos de atención denominados como medicina gerenciada, donde se hacen necesarias las constantes evaluaciones costo-beneficio, racionalización del gasto y en fin, lo que para muchos ha sido la medicina defensiva donde además el paciente se ha denominado cliente o usuario. 
3. Aparición de la bioética: con todo este panorama no debe extrañarnos que algunos se pusieran a pensar de otra manera, aunque como área del conocimiento es bastante reciente: alrededor de tres décadas. De ese nuevo pensamiento deseo destacar que se deben reconocer los cuatro principios fundamentales que son pilares en el análisis del creciente número de conflictos que aparecen en el ejercicio diario: autonomía o principio de la autodeterminación, beneficencia, no maleficencia o la ética del no daño y justicia o ética de la no discriminación.

Se han enfatizado los derechos de los pacientes entre los que deseo destacar los siguientes: acceso a la atención requerida sin discriminación; conocer quien lo atiende; recibir amplia información incluidos los riesgos y los beneficios; la confidencialidad; decidir su participación en investigaciones incluyendo la posibilidad de retiro de las mismas y autorizar el o los tratamientos propuestos. En suma, a que se le trate con dignidad y respeto.

\section{EI anciano}

Me propongo plantear un panorama general de lo que podría ser la situación de salud de un anciano. Lo primero que hay que resaltar es que todas las decisiones acerca de la calidad de la vida y de la muerte recaen en la propia persona y esto como es natural tiene que ver con el respeto de su autonomía. En segundo lugar hay que tener muy presente la creciente posibilidad de convivencia con diferentes enfermedades ya sean terminales o no, que pueden conllevar la formulación de una gran cantidad de medicamentos. En tercer lugar el riesgo de encontrarse en situación social de exclusión.

\section{Dilemas éticos en la prestación de servicios de salud}

1. Objetivos del tratamiento: es importante tener en cuenta que el objetivo principal en la atención en pacientes ancianos no es solo curar; cada vez entran con mayor vigor las terapias paliativas en los casos de las llamadas enfermedades terminales. Cuando se establece la necesidad de un tratamiento, este siempre debe estar mediado por los parámetros de bienestar del propio paciente, mientras que el prestador de salud deberá ajustar estas necesidades buscando la mayor funcionalidad no solo física sino los aspectos psicológicos, sociales y espirituales.

\section{Relación médico-paciente (personal de la salud-} anciano): como se ha mencionado, esta interacción debe estar enmarcada siempre dentro del parámetro del respeto por la autonomía, es decir de la dignidad de la persona. Es muy deseable una actitud amplia de entendimiento por parte del prestador, mejorando así los canales de comunicación, es decir de información y de escucha, que tengan como resultado procurar las decisiones autónomas del anciano y por supuesto una mejor calidad del servicio.

Una situación particularmente sensible es la edad como factor de discriminación; ya se ha mencionado la tácita ecuación de vejez con enfermedad; de ello también se ha podido comprobar la frecuente idea de que no vale la pena gastar dinero en tratar enfermedades costosas en los ancianos. Otra manera de discriminación es que no se invierte en investigaciones como en otros grupos de edad, esto sumado a la visión utilitarista de que el anciano ya no es productivo.

3. Capacidad de decisión: es claro que en la medida que avanza la edad se van disminuyendo la memoria, la atención o las capacidades sensoriales; esto es probable que termine en un aislamiento cada vez mayor. Sin embargo, esta condición no necesariamente impide siempre la capacidad de decidir, comunicar, aceptar o rechazar un tratamiento. Es claro que en la medida que las decisiones sean más trascendentales, por ejemplo que involucren la propia vida, se exigirá un mayor grado de competencia mental que para decisiones de menor complejidad como la de cambiar de sitio de vivienda.

Esta capacidad de decisión marca el grado de competencia de la persona, el cual puede tener además efectos legales como es la interdicción judicial. De otra parte, también se deberán tomar las acciones 
en el caso de las decisiones por sustituto, es decir cuando esa persona es incompetente para tomar una determinada acción, un tercero lo hará siguiendo una de las siguientes premisas: 1) el criterio de la autonomía pura: el que tomaría la persona previamente autónoma que en algún momento manifestó una decisión o preferencia relevante; 2) los juicios sustitutivos: la decisión debería ser aquella que la persona incompetente tomaría en caso de ser competente y 3) el criterio del mejor interés del paciente: optar por el mayor beneficio que el enfermo podría obtener entre todas las opciones posibles.

Algunas situaciones que siempre deben ser analizadas en cuanto a decisiones anticipadas son la institucionalización en los denominados ancianatos o el traslado desde el hospital hasta un hospice o el hogar, en caso de que se requieran cuidados paliativos. Es necesario mencionar los grandes conflictos que se presentan en los denominados pacientes terminales: la utilización o no de tratamientos que de acuerdo con el grado avanzado de evolución de una enfermedad se considerarían ya inútiles (futilidad terapéutica); el inicio o no de medicaciones cada vez más complejas con dudas de que mejoren una condición clínica grave e irreversible y que es probable que retrasen en forma injustificada la muerte con un mayor sufrimiento (pendiente resbaladiza, ensañamiento terapéutico, distanasia); también la denominada eutanasia pasiva o la utilización por ejemplo, de fármacos opiáceos (principio del doble efecto).

\section{Ética de la vejez}

Una de las metas es evitar la muerte prematura de los ancianos, es decir, aquella que sucedería antes de que termine el período natural de la vida de esa persona, que debe ir acompañada del hecho de prevenir o mejorar el sufrimiento. Pero se hace necesario decir también que no es ético retrasar de manera injustificada la muerte, cuando conlleve un aumento del padecimiento de dolores o de malestar tan severos que a juicio del paciente prolongar ese tipo de vida es peor que su propia muerte. Por esto hay que pensar que antes de medicalizar la vejez, la enfermedad y la muerte, hay que humanizarlas. Cada día somos testigos de más instrumentalización de la muerte en detrimento de lo que se ha conocido como "muerte natural". Es muy frecuente hoy que las personas mueran cada vez más en sitios (hospitales o UCI) y de la forma (sin sus seres queridos) como precisamente no quieren. En resumen, si miramos estas importantes cuestiones bajo los parámetros de los principios universales de la bioética, podemos afirmar lo siguiente:

Autonomía: el anciano puede disponer de su vida y de su muerte; tiene derecho a rechazar toda asistencia, incluso la indicada, aún con competencia disminuida. Beneficencia: se deben utilizar aquellos procedimientos que sean los indicados, con el consentimiento del paciente. No maleficencia: jamás utilizar procedimientos contraindicados, siempre se buscará no hacer daño valorando el balance riesgobeneficio. Justicia: no puede haber ningún tipo de discriminación del anciano, ya sea por la edad o por padecer enfermedades que exigen tratamientos costosos.

\section{Conclusilones}

El fenómeno creciente del incremento de la edad de la población merece una revisión general; desde la bioética se deberán analizar ciertos paradigmas como el de creer que estas personas con edad avanzada son siempre enfermos o el doble sentimiento hacia el anciano como objeto de venerabilidad y desprecio, lo que cuestiona muchos de los valores morales actuales. Un campo especialmente álgido es el de la relación del médico y el personal de salud con el paciente anciano, que como en el resto del ámbito de la prestación de los servicios, se debería reconocer en esta persona un sujeto moral que por supuesto merece ser tratado con dignidad; aunque con alguna limitación de su capacidad para la toma de decisiones, debe respetársele su autonomía; en caso de enfermedad es necesario replantear los objetivos de los tratamientos pues es probable que serán cada vez 
más de tipo paliativo que curativo; siempre se debe realizar el balance riesgo-beneficio; la enfermedad y la muerte forman parte de la vida y en todo caso se debe reconocer su dignidad como persona. Por último, por su edad no pueden ser objeto de ningún tipo de discriminación.

\section{Lecturas recomendadas}

- Beauchamps TL, Childress JF. Principios de ética biomédica. Barcelona: Masson, 1998.

- Del Campo Alepuz G. Exclusión social y sida [monografía en Internet]. Alicante, España : Universidad de Alicante; 2001 [citado 03 Sep 2008]. Disponible en: http://perso. wanadoo.es/aniorte nic/toc.html

- Escobar Triana J. Morir como ejercicio final del derecho a una vida digna. Bogotá : Ediciones El Bosque; 1998.

- Gracia D. Ética de los confines de la vida. Bogotá: El Búho; 2002.
- Kübler-Ross E. Sobre la muerte y los moribundos. Barcelona: Grijalbo; 1974.

- Lolas Stepke F. Bioética y vejez: el proceso de desvalimiento como constructo biográfico. En: Contemporaneidad y bioética. Persona y sociedad. Santiago: Universidad de Chile; 1994.

- Lolas Stepke F. Las dimensiones bioéticas de la vejez. Acta bioeth. 2001; 7(1): 57-70.

- Mercado C. Dilemas bioéticos en geriatría: toma de decisiones médicas. Acta bioeth. 2001; 7(1): 129-41.

- Outomuro D. Algunos dilemas éticos en torno a la vejez. Ars Med. Rev. de Huma [serie en Internet]. 2003 [citado 12 Jun 2008]; 8 (8): [aprox. 7 p.]. Disponible en: http://escuela. med.puc.cl/publ/arsmedica/ArsMedica8/Art05.html.

- Roqué MV, Pérez Guerrero J. Bioética geriátrica. Rev Mult Gerontol. [serie en Internet]. 2002 [citado 12 Jun 2008]; 12(1): [aprox. 5 p.]. Disponible en: http://www. nexusediciones.com/pdf/gero2002_1/g-12-1-004.pdf
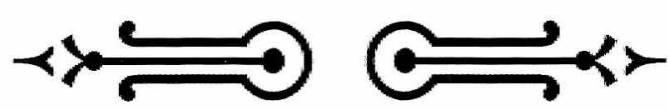